\title{
Carga física, estrés y morbilidad sentida osteomuscular en trabajadores administrativos del sector público
}

Physical load, stress and musculoskeletal morbidity in administrative workers in the public sector

Lesly Castillo-Ante' ${ }^{1}$ orcid.org/0000-0002-1633-1213

Cecilia Ordoñez-Hernández² orcid.org/0000-0001-9296-3768

Andrea Calvo-Soto3* orcid.org/0000-0002-1723-9021

1 Corporación Occidente IPS. Popayán, Colombia

2 Facultad Ciencias de la Salud. Universidad Libre. Cali, Colombia

3 Facultad de Salud. Universidad Santiago de Cali. Cali, Colombia

\section{Resumen}

Introducción: Los desórdenes musculoesqueléticos se asocian entre otros, con la carga física y aspectos organizacionales del trabajo. Objetivo: Determinar la relación entre la carga física, los niveles de estrés y la morbilidad sentida osteomuscular en trabajadores administrativos del sector público de Popayán (Colombia). Materiales y métodos: Estudio cuantitativo, transversal analítico-correlacional. La muestra aleatoria simple fue de 104 trabajadores, los instrumentos de recolección fueron: Evaluación de puesto de trabajo RULA (acrónimo de Rapid Upper Limb Assessment), cuestionario de Maslach y cuestionario nórdico estandarizado (Kuorinka et al., 1987), se realizó análisis uni y bivariado, para la comprobación hipótesis se utilizó el estadístico Chi Cuadrado. Resultados: Se encontró que 76\% de los puestos de trabajo requiere investigación con posible necesidad de cambios. El 79\% de los trabajadores tuvo un nivel bajo de cansancio y $83 \%$ de despersonalización. El dolor más prevalente en los últimos seis meses fue de cuello (51\%), seguido de espalda (36\%) y hombro (33\%). Se encontró correlación estadísticamente significativa entre la carga postural y presencia del dolor muscular $(p=0,301)$. Conclusión: La carga postural aumentada está relacionada con la presencia de dolor de cuello, sin embargo, el dolor no está relacionado con la presencia de estrés laboral.

Palabras clave: Salud laboral; ergonomía; estrés laboral; carga de trabajo. (Fuente: DeCS, Bireme).

\begin{abstract}
Introduction: Musculoskeletal disorders are associated with physical and organizational aspects of work, among others. Objective: To determine the relationship between physical load, levels of stress, and musculoskeletal morbidity in administrative workers in the public sector of Popayán (Colombia), between 2016 and 2017. Materials and methods: An analytical-correlational, cross-sectional, quantitative study of a random sample of 104 workers. The data collection instruments were: the Rapid Upper Limb Assessment (RULA), the Maslach Burnout Inventory (MBI) and the Standardized Nordic Questionnaire (Kuorinka et al., 1987). Uni- and bivariate analysis were conducted, as well as a Chi-square to test the hypothesis. Results: It was found that $76 \%$ of the workplaces require further research and need changes. $79 \%$ of employees experienced low fatigue levels, whereas $83 \%$ manifested depersonalization. During the last six months, the most common pains reported were neck pain (51\%), followed by back pain (36\%) and shoulder pain (33\%). There was a statistically significant correlation between postural load and muscle pain $(p=0.301)$. Conclusion: Although an increased postural load is related to neck pain, this pain is not associated with workplace stress.
\end{abstract}

Key words: Occupational health; ergonomics; occupational stress; workload. (Source: DeCS, Bireme). 


\section{Introducción}

Los trastornos musculoesqueléticos (TME) se encuentran entre los problemas más importantes de salud en el trabajo, tanto en países desarrollados como en vía de desarrollo. En Colombia son las patologías más comunes entre los trabajadores, en 2011 estuvieron entre $2 \%$ y $10 \%$ de las enfermedades de origen laboral, los diagnósticos más frecuentemente reportados fueron: lumbago no específico, epicondilitis lateral, síndrome de manguito rotador y síndrome de túnel carpiano(1).

Respeto al impacto económico, en 2015 en Gran Bretaña los TME en conjunto con el estrés y la depresión/ansiedad, representaron la mayoría de los días perdidos debido a problemas de salud relacionados con el trabajo(2), en Estados Unidos en 2007 los costos directos de los TME y síndrome del túnel carpiano fueron de 1,5 y 0,1 mil millones de dólares y los indirectos de 1,1 y 0,1 mil millones ${ }^{(3)}$ y en Colombia en 2013 el gasto por estas enfermedades ascendió a $\$ 44$ mil millones, 15,3\% más que en 2012(4). Los TME son entonces una importante condición que atender, no sólo por la identificación de las condiciones de trabajo que los generan o las consecuencias que producen sobre la salud, sino también por el impacto económico para las empresas y el sector de la salud, hecho que justifica las acciones y la intervención con perspectiva preventiva.

Regularmente los TME se han relacionado con actividades desarrolladas en el sector manufacturero; sin embargo, es importante analizar otros sectores como el de servicios, en particular las tareas asignadas al personal administrativo, donde se ha reportado mayor riesgo de padecer TME en miembros superiores y espalda (5-7) como: cervicalgia, dorsalgia, lumbalgia y síndrome del túnel carpiano, provocando una cifra considerable de incapacidades(8-10).

Existen otros factores no biomecánicos relacionados con el desarrollo de TME, como son las condiciones salariales(11) o la tensión por el trabajo, el estrés laboral, el estrés psicosocial y la fatiga(12). Jacukowicz encontró que la posibilidad de presentar síntomas musculoesqueléticos en la zona del cuello aumenta a medida que el entorno físico empeora, hay presencia de conflictos interpersonales y se incrementa el estrés en la organización(12). Asimismo, existe correlación entre la intensidad del dolor, el dolor lumbar, de hombro y muñeca con la baja satisfacción en el trabajo ${ }^{(13,14)}$ y se han determinado los riesgos psicosociales y el estrés como predictores de dolor musculoesquelético(15).

El Instituto Nacional de Salud y Seguridad Ocupacional (NIOSH) de Estados Unidos, establece tres posturas teóricas generales sobre la relación entre los TME y los factores psicosociales en el trabajo: (1) las demandas psicosociales y el estrés del trabajo pueden producir incremento de la tensión muscular y exacerbar el esfuerzo biomecánico relacionado con la tarea; (2) las demandas psicosociales pueden afectar la conciencia reportando síntomas musculoesqueléticos o también afectan las percepciones debido a esta causa, y (3) la asociación pueden estar dada por la relación causal o correlación entre las demandas psicosociales y físicas(16).

Esta relación entre los TME y los factores psicosociales en los trabajos de oficina, se integra además del componente biomecánico y psicosocial, al cognitivo, siendo éste un componente que utiliza la tecnología, su naturaleza implica un trabajo automatizado y mecanizado de información, al cual se le suman los procesos interpretativos del trabajador, influenciados por el contexto y los factores experienciales. Detrás de esta percepción y atribución de síntomas, está la tensión psicológica por los factores individuales y de la organización del trabajo, por ejemplo, el clima laboral influye directamente en cómo los trabajadores detectan, interpretan y responden a las demandas físicas(16).

Aunque existen estudios internacionales sobre este tema, son pocos los estudios en América Latina que relacionen la etiología de los dolores musculares con los factores psicolaborales. El objetivo del estudio fue relacionar la carga física, niveles de estrés y morbilidad sentida osteomuscular en trabajadores administrativos del sector público de PopayánColombia.

\section{Materiales y métodos}

Se realizó un estudio cuantitativo, transversal, correlacional. La población estuvo conformada por 160 trabajadores administrativos de una institución pública de Popayán. Se realizó muestreo aleatorio simple, se calculó un tamaño muestral de 104 
personas considerando nivel de confianza de 95\% y error relativo de 5\%. Los criterios de inclusión fueron ser trabajador administrativo con antigüedad mayor a seis meses y el deseo de participar voluntariamente en la investigación. Los criterios de exclusión fueron tener diagnóstico médico de patologías osteomusculares y trastornos psicológicos o depresivos previos al estudio. Se aplicaron simultáneamente los cuestionarios Nórdico y Maslash(17-21), una vez diligenciados se procedió a la valoración del puesto de trabajo con el método RULA (acrónimo de Rapid Upper Limb Assessment) a través de observación detallada(22).

El Cuestionario Nórdico es una herramienta utilizada para valorar la sintomatología dolorosa de origen musculoesquelético que consta de 45 ítems que indagan por los síntomas en diferentes segmentos corporales: cuello, hombro, codo, mano, espalda alta, baja, cadera, rodilla, tobillo y pie(17). Las preguntas son autoadministrables de selección múltiple. Se utilizó la versión validada para población española con coeficientes de consistencia y fiabilidad entre 0,727 y $0,816^{(18,19)}$, no existe una validación para población colombiana.

El cuestionario de Maslach Burnout Inventory(20) consta de 22 ítems, que miden el nivel de estrés a partir de las dimensiones: cansancio emocional, despersonalización y realización personal. El instrumento fue validado para población colombiana por Oramas et al.,(21) con un índice de confiabilidad de 0,711 .

El método RULA desarrollado en 1993 por McAtamney y Corlett, evalua la exposición de los trabajadores a factores de riesgo que originan una elevada carga postural y que pueden ocasionar trastornos en los miembros superiores del cuerpo. El método debe ser aplicado al lado derecho y al lado izquierdo del cuerpo por separado(22); en esta investigación no se realizó ninguna adaptación al método. Se consideraron las variables edad, género, escolaridad, práctica de deporte, antigüedad en el cargo, horas laborales por día, peso, talla, índice de masa corporal, tipo de contratación, número de personas a cargo, morbilidad sentida, intervención recomendada por RULA, agotamiento, despersonalización, baja realización personal.

Las variables cualitativas fueron descritas, las cuantitativas se analizaron con el programa estadístico SPSS. Para la comprobación de las hipótesis se utilizó el estadístico de Chi cuadrado considerando un $\alpha<0,05$.

\section{Consideraciones éticas}

Esta investigación se acogió a los acuerdos internacionales de investigación biomédica según el acuerdo de Helsinki. Se considera una investigación "sin riesgo" según el artículo 11, numeral "a" de la Resolución 8430 de 1993 del Ministerio de Salud y Protección Social de Colombia.

La investigación contó con el aval de Comité de Ética de la Universidad Libre seccional Cali. Se garantizó la estricta confidencialidad de los datos a través de la encriptación de la información de identificación con códigos numéricos, los participantes fueron tratados con respeto y dignidad al igual que su información. Todas las personas que cumplieron con los criterios de inclusión tuvieron la oportunidad de participar sin ningún tipo de discriminación, así como la opción de retirarse en cualquier momento si así lo consideraran, según lo definido en el consentimiento informado utilizado. Por el diseño de investigación, este estudio no representó un riesgo para la salud de los participantes, no generó ninguna contraprestación ni afectación a la integridad del ambiente.

\section{Resultados}

La población participante en su mayoría fue de género femenino (66\%), el rango de edad estuvo entre 23 y 58 años con una media de $39.4 \pm 10.3$ años, el $56,7 \%$ declaró no practicar ningún deporte y $37 \%$ se encontró en sobrepeso. El 45\% manifestó estar soltero, $45 \%$ tener formación profesional, $54,8 \%$ estar contratado por prestación de servicios y trabaja entre 6 y 15 horas por día, 58\% tiene una antigüedad laboral en la empresa entre 1 y 5 años.

Se reportó dolor del cuello (51\%), espalda (36\%) y hombro (33\%) en los últimos seis meses, gradiente que se mantuvo en los últimos 7 días $(36 \%, 27 \%$ y $25 \%$ respectivamente). El dolor de cuello (18,3\%) y de la mano y muñeca $(15,4 \%)$ fueron los que provocaron mayores dificultades para realizar el trabajo y restringieron las actividades de la vida diaria $(13,8 \%$ y $14,4 \%$ respectivamente) (Tabla 1$)$. 
Tabla 1. Prevalencia de dolor por región anatómica y disminución de actividades laborales y extralaborales por dolor en trabajadores administrativos del sector público de Popayán

\begin{tabular}{|c|c|c|c|c|c|c|c|c|}
\hline \multirow[t]{2}{*}{ Región anatómica } & \multicolumn{2}{|c|}{$\begin{array}{c}\text { Dolor en los últimos } \\
\text { seis meses }\end{array}$} & \multicolumn{2}{|c|}{$\begin{array}{c}\text { Dolor en los } \\
\text { últimos siete días }\end{array}$} & \multicolumn{2}{|c|}{$\begin{array}{l}\text { Dificultad para } \\
\text { trabajar por dolor }\end{array}$} & \multicolumn{2}{|c|}{$\begin{array}{l}\text { Disminución de actividades por } \\
\text { dolor }\end{array}$} \\
\hline & $\mathbf{n}$ & $\%$ & $\mathbf{n}$ & $\%$ & $\mathbf{n}$ & $\%$ & $\mathbf{n}$ & $\%$ \\
\hline Cuello & 51 & 49 & 36 & 34,6 & 19 & 18,3 & 14,4 & 13,8 \\
\hline Hombro & 33 & 31,7 & 25 & 24 & 14 & 13,5 & 14,4 & 13,8 \\
\hline Codo & 10,6 & 10,2 & 7 & 6,7 & 5 & 4,8 & 4 & 3,8 \\
\hline Mano/Muñeca & 29 & 27,9 & 21 & 20,2 & 16 & 15,4 & 15 & 14,4 \\
\hline Espalda & 36 & 34,6 & 27 & 26 & 12,5 & 12 & 12,5 & 12 \\
\hline Cadera & 4 & 3,8 & 3 & 2,9 & 2 & 1,9 & 3 & 2,9 \\
\hline Rodilla & 4 & 3,8 & 96 & 92,3 & 0 & 0 & 1 & 1 \\
\hline Tobillo & 2 & 1,9 & 0 & 0 & 1 & 1 & 1 & 1 \\
\hline
\end{tabular}

En cuanto a la intensidad del dolor, los de cuello, hombro y mano fueron calificados como moderados $23 \%, 12,5 \%$ y $11,5 \%$ respectivamente.

El 16\% presentó dolor en cuello y 10\% en espalda en un rango de tiempo entre 13 meses a 3 años, el $12 \%$ presentó dolor de hombro y $11 \%$ de espalda, en un rango de 2 a 6 meses. El 19,2\% consultaron al médico por dolor de cuello, 17,3\% por dolor de hombro y $12,5 \%$ por dolor de espalda. El 32\% de los empleados atribuyó su dolor al estrés, $24 \%$ al movimiento repetitivo el $21 \%$ y $21 \%$ a la postura.

En relación a los niveles de estrés, valorados con la escala de Malasch, 79\% de los trabajadores está en un nivel bajo de cansancio; $83 \%$ en un nivel bajo de despersonalización y $73 \%$ en nivel alto de realización personal (Tabla 2).

Tabla 2. Niveles de estrés en trabajadores administrativos del sector público de Popayán

\begin{tabular}{lrrlllrr}
\hline \multirow{1}{*}{ Variable } & \multicolumn{2}{c}{ Bajo } & \multicolumn{2}{c}{ Medio } & \multicolumn{2}{c}{ Alto } \\
\cline { 2 - 7 } & n & $\mathbf{\%}$ & n & \% & \multicolumn{1}{c}{ n } & \multicolumn{1}{c}{$\%$} \\
\hline Cansancio & 82 & 79 & 18 & 17 & 4 & 4 \\
Despersonalización & 86 & 83 & 13 & 12 & 5 & 5 \\
Realización Personal & 8 & 8 & 20 & 19 & 76 & 73 \\
\hline
\end{tabular}

Con relación a la carga postural medida por RULA el $76 \%$ de los puestos tiene un nivel de riesgo medio de TME y requiere investigación con posible necesidad de cambios, el 13\% tiene un nivel de riesgo muy alto de TME que demanda análisis con cambios rápidos, un $11 \%$ con una postura aceptable.

Al realizar el cruce de variables, se encontró significancia estadística para la relación entre: género $\mathrm{y}$ despersonalización $(p=0,031)$, dolor de hombro y género $(p=0,049)$, dolor en la mano $\mathrm{y}$ práctica de deporte $(p=0,003)$, dolor de espalda y años en el cargo $(p=0,010)$, dolor de cuello y carga postural $(p=0,003)$, dolor de codo y cansancio emocional $(p=0,037)$ (Tabla 3).

Durante la aplicación de los instrumentos se identificó que había falta de actualización del mobiliario y mantenimiento, así como disposición inadecuada del mobiliario con relación a los espacios y las fuentes de luz, también ausencia de inducción en el ajuste y uso correcto de los materiales y herramientas de trabajo.

Tabla 3. Relación entre la carga postural y niveles de estrés con presencia de dolor por región anatómica

\begin{tabular}{|c|c|c|c|c|c|c|c|c|}
\hline Variables de contraste & Cuello & Hombro & Codo & Mano & Espalda & Cadera & Rodilla & Tobillo \\
\hline Carga Postural & $0,003^{*}$ & 0,685 & 0,228 & 0,409 & 0,543 & 0,483 & 0,483 & 0,623 \\
\hline Cansancio Emocional & 0,390 & 0,679 & $0,037^{*}$ & 0,160 & 0,156 & 0,291 & 0,848 & 0,460 \\
\hline Despersonalización & 0,143 & 0,538 & 0,356 & 0,644 & 0,447 & 0,678 & 0,078 & 0,217 \\
\hline Realización Personal & 0,497 & 0,629 & 0,854 & 0,169 & 0,515 & 0,556 & 0,556 & 0,680 \\
\hline
\end{tabular}

${ }^{*} p<0,05$, estadísticamente significativo.

\section{Discusión}

Respecto a la morbilidad sentida osteomuscular, las zonas corporales que presentaron mayor prevalencia de dolor fueron el cuello, hombros, espalda y muñecas, tal como lo reportado por otros estudios, en los cuales se ha demostrado que el rol ocupacional del trabajo administrativo está relacionado con el riesgo por carga física, dado que requiere de posturas prolongadas en posición sedente, exige activación de la musculatura de la espalda y todo el tren superior incluido el cuello, 
movimientos repetidos de hombro y muñeca, que aumentan la probabilidad de aparición de sintomatología dolorosa(23,24); además prevalece la posición sentada excesiva, que se asocia con la presencia de síntomas musculo esqueléticos en miembros superiores, espalda baja y miembros inferiores(25). Situación que empeora a medida que aumentan tanto la edad como la exposición(26,27), se encontró en este caso relación entre la antigüedad en el cargo y la presencia de dolor en la espalda.

Por el tipo de actividades que ejecutan los trabajadores frente a portales de computador, realizan poca actividad física, presentan altos índices de sedentarismo, sobrepeso u obesidad, aumentando el nivel de fatiga muscular y dolor(26,27); estas molestias afectan las actividades tanto laborales como extralaborales(16,28).

Se encontró carga postural aumentada, el 76\% requiere investigación y posible necesidad de cambios, el 13\% requiere cambios rápidos. Este trabajo encontró correlación significativa entre el dolor en cuello y la carga postural, cuando en un puesto de trabajo se desarrollan posturas inadecuadas se presenta sobrecarga y fatiga de ciertos grupos musculares, generando una alteración en la biomecánica del cuerpo, dando paso a la aparición de dolores musculares, estos hallazgos coinciden con lo referido por Mendinueta y Herazo, quienes mencionan que tener posiciones prolongadas y soportar cargas inadecuadas, influye en la presencia de desórdenes osteomusculares en miembros superiores ${ }^{(29)}$.

Es importante señalar, que al ser una institución de carácter público existe falta de actualización de mobiliario, la combinación de muebles equipos y materiales que se han obtenido en diferentes administraciones, la falta de mantenimiento, la disposición inadecuada del mobiliario con relación a los espacios y las fuentes de luz, así como la ausencia de inducción en el ajuste y uso correcto de los materiales y herramientas de trabajo, aumentan la exposición por falta de confort y empeora el riesgo por carga física(30). El hallazgo de la carga postural aumentada refleja la necesidad de cambios en los puestos de trabajo, readaptación, mejoramiento ergonómico, y la inclusión de procesos educativos de autocuidado, como las buenas prácticas de higiene postural (30).
El tipo de responsabilidades que se asume en estos cargos público-administrativos, podrían generar altos niveles de estrés, pero en este caso el análisis de la variable estrés evidenció que la mayoría de los trabajadores están en nivel bajo de cansancio emocional, despersonalización y alto nivel de realización personal, lo cual contrasta con lo encontrado por Eijckelhofab et al., en cuanto al estrés percibido(31). Los resultados encontrados se pueden asociar con el hecho de que la población estudiada tiene niveles altos de satisfacción personal que puede actuar como un factor protector, a mayor nivel de satisfacción en el trabajo se aminora el efecto negativo sobre la salud(32).

A pesar de que se cree que la falta de seguridad laboral es un fuerte predictor de mala salud, por las nuevas formas de contratación como la prestación de servicios (33), en este estudio no hubo altos niveles de estrés percibido, pese a que la mitad de los participantes tienen este tipo de contrato; los resultados pueden deberse a que el tipo de contratación no predice la felicidad en el trabajo, el optimismo y el pesimismo laborales, evidenciando así que influyen aspectos estructurales, más estables, que no dependen de condiciones laborales o circunstancias externas ${ }^{(34)}$.

Este es un aspecto que refleja como el crecimiento individual, el ambiente laboral positivo, el tipo de contratación, los bajos niveles de estrés, permiten al trabajador afrontar de manera adecuada las exigencias de su quehacer laboral, protegiéndolo de estrés y dolores musculares, pues a mayor nivel de estrés existe más fatiga muscular(35).

\section{Conclusiones}

En la población de trabajadores administrativos del sector público de Popayán, se identificó la necesidad de realizar un reenfoque ergonómico en los puestos de trabajo y educación para promover conductas de autocuidado que disminuyan el riesgo biomecánico.

Considerando que los niveles altos de realización personal son un factor protector de sintomatología osteomuscular, las empresas deben enfocarse en trabajar con sus empleados estos aspectos, propiciar oportunidades laborales que enriquezcan el crecimiento y la satisfacción tanto laboral como personal, como una medida para mantener la salud física y mental del personal. 
Esta investigación aporta al análisis del riesgo biomecánico, contextualizándolo dentro de los aspectos psicosociales, priorizando que para mantener bienestar físico debe ligarse siempre la esfera mental y social, pues apartar estos aspectos hace que se pierda el entendimiento completo del fenómeno.

\section{Recomendaciones}

Para futuros estudios se recomienda el uso de software para el análisis de la carga física que agilicen el procesamiento de información y permitan ampliar la población de estudio.

Conflicto de intereses: Los autores no manifiestan conflicto de intereses.

\section{Referencias}

1. Federación de aseguradores colombianos. Estadísticas Presidenciales Ministerio de Salud y Protección Social Sistema de Información Gremial. Bogotá; 2013. Disponible en: $\quad$ https://fasecolda.com/cms/wpcontent/uploads/2019/08/enfermedad-laboral-colombia2013.pdf

2. Health and Safety Executive. Health and safety statistics Annual Report for Great Britain. Summary Statistics. Health and Safety Executive; 2015. Disponible en: https://www.hse.gov.uk/statistics/index.htm

3. Bhattacharya A. Costs of occupational musculoskeletal disorders (MSDs) in the United States. Int J Ind Ergon. 2014; 44(3): $448 \quad-\quad 454.4$ DOI: http://dx.doi.org/10.1016/j.ergon.2014.01.008.

4. Ministerio del trabajo. Segunda Encuesta Nacional de Condiciones de Salud y Trabajo. Santa Fé de Bogotá. 2013. Disponible en: https://fasecolda.com/cms/wpcontent/uploads/2019/08/ii-encuesta-nacional-seguridadsalud-trabajo-2013.pdf

5. Van Eerd D, Munhall C, Irvin E, Rempel D, Brewer S, van der Beek AJ, et al. Effectiveness of workplace interventions in the prevention of upper extremity musculoskeletal disorders and symptoms: an update of the evidence. Occup Environ Med. 2016; 73(1):62- 70. doi: 10.1136/oemed2015-102992 20. Disponible en: https://www.ncbi.nlm.nih.gov/pubmed/26552695

6. Petit A, Ha C, Bodin J, Rigouin P, Descatha A, Brunet R, et al. Risk factors for carpal tunnel syndrome related to the work organization: A prospective surveillance study in a large working population. Applied Ergonomics. 2015; 47: 51-10. doi:10.1016/j.apergo.2014.08.007

7. Instituto Nacional de Seguridad e Higiene en el Trabajo. El trastorno musculoesquéletico en el ámbito laboral en cifras Departamento de Información e Investigación del Instituto Nacional de Seguridad e Higiene en el Trabajo. MEYSS; 2012.Disponible

en: https://www.insst.es/documents/94886/96076/El+trastor no+musculoesquel $\% \mathrm{C} 3 \% \mathrm{~A} 9$ tico+en+el+\%C3\%A1mbito+lab oral+en+cifras/0e803148-d396-4ba8-ab49-6b9a5dc8726a
8. Mendinueta-Martínez M, Herazo-Beltrán Y. Percepción de molestias musculoesqueléticas y riesgo postural en trabajadores de una institución de educación superior. Salud Uninorte. Barranquilla. 2014; 30 (2): 170-179. Disponible en: http://rcientificas.uninorte.edu.co/index.php/salud/article /viewFile/5324/6997

9. López-Torres B, González-Muñoz E, Colunga-Rodríguez C, Oliva-López E. Evaluación de Sobrecarga Postural en Trabajadores: Revisión de la Literatura. Cienc Trab. 2014;16(50): 111-115. Disponible en: https://scielo.conicyt.cl/scielo.php?script=sci_arttext\&pid= S0718-24492014000200009

10. Loghmani A, Golshiri P, Zamani A, Kheirmand M, Jafari N. Musculoskeletal symptoms and job satisfaction among office-workers: a cross-sectional study from Iran. Acta Médica Académica. 2013; 42(1):46-54. doi: 10.5644/ama2006-124.70

11. Nawawi R, Deros B, Daruis D, Ramli A, Zein R, Joseph L. Effects of payment method on work control, work risk and work-related musculoskeletal health among sewing machine operators. Journal of Mechanical Engineering and Sciences. 2015; 9: 1705-1713. Doi: 10.15282/jmes.9.2015.16.0164

12. Jacukowicz A. Psychosocial work aspects, stress and musculoskeletal pain among musicians. A systematic review in search of correlates and predictors of playing-related pain. Journal: Work. 2016; 54(3):657-68. Doi: 10.3233/WOR-162323.

13. Herr R, Bosch J, Loerbroks A, Van Vianen A, Jarczok M, Fischer J, Schmidt B. Three job stress models and their relationship with musculoskeletal pain in blue and whitecollar workers. J Psychosom Res. 2015; 79(5):340-47. Doi: 10.1016/j.jpsychores.2015.08.001.

14. Sarkar K, Dev S, Das T, Chakrabarty S, Gangopadhyay S. Examination of postures and frequency of musculoskeletal disorders among manual workers in Calcutta, India. Int J Occup Environ Health. 2016; 22(2):151-158. Disponible en: https://www.ncbi.nlm.nih.gov/pmc/articles/PMC4984967/

15. Márquez-Gómez M, Márquez-Robledo M. Factores de riesgo biomecánicos y psicosociales presentes en la industria venezolana de la carne. Ciencia \& Trabajo. 2015;17(54): 171-176. Disponible en: https://scielo.conicyt.cl/pdf/cyt/v17n54/art03.pdf

16. Sauter S, Moon S. An ecological model of musculoskeletal disorders in office work. In: MoonS, Sauter SL, editors. Psychosocial Factors and Musculoskeletal Disorders in Office Work. Ohio: Beyond Biomechanics; 2014. Disponible en: https://www.cdc.gov/niosh/nioshtic-2/20027620.html

17. Kuorinka I, Jonsson B, Kilbom A, Vinterberg H, BieringSorensen F, Andersson G, et al. Standardised Nordic questionnaires for the analysis of rnusculoskeletal symptoms. Appl Ergon. 1987; 18(3):233-7. Disponible en: http://www.ncbi.nlm.nih.gov/pubmed/15676628

18. Martínez B, Santo S, Bolea M, Casalod Y, Andres E. Validación del cuestionario nórdico musculo-esquelético estandarizado en población española [Internet]. 12th International Conference on Occupational Risk Prevention; 21, 22 y 23 de mayo de 2014; Zaragoza España. Barcelona. Universitat Politècnica de Catalunya. Disponible en: http://www.prevencionintegral.com/en/canalorp/papers/orp-2014/validacion-cuestionario nordicomusculoesqueletico-estandarizado-enpoblacionespanola. 
19. Martínez M, Alvarado R. Validación del cuestionario nórdico estandarizado de síntomas musculoesqueléticos para la población trabajadora chilena, adicionando una escala de dolor. Revista de Salud Pública.2017; 21 (2):41-51. Disponible en: https://revistas.unc.edu.ar/index.php/RSD/article/view/1 6889

20. Oramas A, Gonzales A, Vergara, A. El desgaste profesional. Evaluación y factorización del MBI-GS. Revista cubana de salud y trabajo, 2007; 8 (1): 37-45. Disponible en: http://www.sld.cu/galerias/pdf/sitios/insat/rst06107.pdf

21. Guevara L, Ocampo N. Propiedades psicométricas de confiabilidad y validez del Maslach Burnout Inventory General Survey. Rev. Interam. Psicol. Ocup. 2014; 33 (2):128-142. Disponible en: https://pdfs.semanticscholar.org/c512/45bdac4cddf2349f 23f8935986668dedb44d.pdf

22. McAtamney L, Corlett N, RULA: a survey method for the investigation of world-related upper limb disorders. Applied Ergonomics. 1993; 24 (2):91-99. Disponible en: https://www.ncbi.nlm.nih.gov/pubmed/15676903

23. Vernaza-Pinzón, P., \& Sierra-Torres, C. H. (2005). Dolor músculo-esquelético y su asociación con factores de riesgo ergonómicos, en trabajadores administrativos. Revista de salud pública, 7 (3), 317-326. Disponible en: http://www.scielo.org.co/scielo.php?script=sci_abstract\&pi $\mathrm{d}=\mathrm{S} 0124-00642005000300007$

24. Vieira ER, Kumar S. Working postures: a literature review. Journal of Occupational Rehabilitation. 2004; 14(2):143-59. Disponible https://www.ncbi.nlm.nih.gov/pubmed/15074366

25. Coenen P, Healy GN, Winkler E, Dunstan DW, Owen N, Moodie M, LaMontagne AD, Eakin EA, O'Sullivan PB, Straker LM. Associations of office workers' objectively assessed occupational sitting, standing and stepping time with musculoskeletal symptoms. Ergonomics. 2018 Sep; 61(9):1187-1195. doi: 10.1080/00140139.2018.1462891

26. Merlino L, Rosecrance JC, Anton D, Cook TM. Symptoms of musculoskeletal disorders among apprentice construction workers. Appl Occup Environ Hyg 2003; 18: 57-64. DOI: 10.1080/10473220301391

27. Lebouf-Y de C. Body weight and low back pain. Spine 2000; 25: 226-237. DOI: 10.1097/00007632-200001150-00015

28. Loghmani A, Golshiri P, Zamani A, Kheirmand M, Jafari N. Musculoskeletal symptoms and job satisfaction among office-workers: a cross-sectional study from Iran. Acta
Médica Académica. 2013; 42(1):46-54. doi: 10.5644/ama2006-124.70

29. Mendinueta-Martínez M, Herazo-Beltrán Y. Percepción de molestias musculoesqueléticas y riesgo postural en trabajadores de una institución de educación superior. Salud Uninorte. Barranquilla. 2014; 30 (2): 170-179. Disponible en: http://rcientificas.uninorte.edu.co/index.php/salud/article /viewFile/5324/6997

30. Martínez Moreno P, Aguirre Alemán M, González José, W. Estudio ergonómico como parte de la responsabilidad social en trabajadores del centro regional de informática de la Universidad Veracruzana. Inquietud Empresarial.2015; 15(2), 87-114. Disponible: https://revistas.uptc.edu.co/index.php/inquietud_empresar ial/article/view/7611

31. Eijckelhofab B. Huysmansa, Blatterb B, Leidera P, Johnsond P, DieënbeJack J, Dennerlein J, Beek A. Office workers' computer use patterns are associated with workplace stressors. Applied Ergonomics. 2014; 45(6): 1660-1667. DOI: 10.1016/j.apergo.2014.05.013

32. Di Marco D, López-Cabrera R, Arenas A, Giorgi G, Arcangeli G, Mucci N. Approaching the discriminatory work environment as stressor: the protective role of job satisfaction on health. Frontiers in psychology. 2016; 30(7):1313. doi: 10.3389/fpsyg.2016.01313.

33. Ramaci T, Pellerone M, Ledda C, Rapisarda V. Health promotion, psychological distress, and disease prevention in the workplace: a cross-sectional study of Italian adults. Risk Manag Healthc Policy. 2017; 14(10):167-175. doi: 10.2147/RMHP.S139756.

34. Posada A, Velásquez A, Granada B. Los efectos del tipo de contrato laboral sobre la felicidad en el trabajo, el optimismo y el pesimismo laborales. Revista Interamericana de Psicología Ocupacional. 2016; 34:9-21. Disponible en: https://www.researchgate.net/profile/Alejandro_Posada2/ publication/311751101_Los_efectos_del_tipo_de_contrato_l aboral_sobre_la_felicidad_en_el_trabajo_el_optimismo_y_el_p esimismo_laborales/links/5a58aa46a6fdcc80fdf000ca/Losefectos-del-tipo-de-contrato-laboral-sobre-la-felicidad-enel-trabajo-el-optimismo-y-el-pesimismo-laborales.pdf36

35. Bougea A, Kleisarchakis $M$, Spantideas N, Voskou $P$, Thomaides T, Chrousos G, Belegri S. Job stress and burnout in the care staff of Leros PIKPA Asylum 25 years after the first Deinstitutionalisation and Rehabilitation Greek Project. BJPsych Bull. 2016; 40(6): 296-301. doi:10.1192/pb.bp.115.052258 